\title{
EDITORIAL
}

\section{Cannabinoids in glioblastoma multiforme-hype or hope?}

Cannabis and its derivatives are being used increasingly by patients with cancer, including patients with glioblastoma multiforme (GBM), the most common and aggressive primary brain malignancy. Despite promising preclinical data suggesting potential anticancer effects for cannabinoids in GBM, clinical and safety data are lacking. This editorial will discuss a recent Phase $1 \mathrm{~b}$ trial of nabiximols oromucosal spray in combination with dose-intense temozolomide in patients with recurrent GBM in the context of other relevant findings in this field.

British Journal of Cancer https://doi.org/10.1038/s41416-021-01265-5

Cannabinoids are diverse compounds that are ligands for endogenous cannabinoid and other G-protein coupled receptors. $^{1-3}$ Some of these are being investigated increasingly in preclinical models and clinical studies in a variety of cancers, assessing their potential in ameliorating symptoms or for anticancer effects. The group comprises plant-derived phytocannabinoids (including cannabidiol (CBD) and the psychoactive $\Delta^{9}$ tetrahydrocannabinol (THC)), synthetic substances (including nabilone and dronabinol which are FDA-approved for chemotherapy-induced nausea and vomiting), and a variety of (physiological) endocannabinoids.

While the use of cannabis grown specifically for 'medical purposes', and its extracts (e.g. CBD oil), by cancer patients has increased significantly in recent years, high-quality evidence supporting their safety and utility is sparse, and products comprise inconsistent ingredients with inconsistent dosage. Although reports suggest dysregulation of cannabinoid receptors and their endogenous ligands in cancer, results to date have generally been inconclusive and suggest both pro-tumorigenic and anti-cancer effects. ${ }^{1-3}$ It is unclear whether these inconsistencies reflect an incomplete understanding of this complex biological system, heterogeneity in the function of ligated receptors in distinct tissues or disease states, or bystander effects (where changes in this system are not central to cancer progression or maintenance). Nevertheless, multiple preclinical models do support a role for modulation of cannabinoid and other receptors (through genetic or pharmacological manipulation) in affecting tumour cell proliferation, survival and invasion, and the tumour microenvironment, including immune and endothelial cells.

Glioblastoma multiforme (GBM) is the most common, and most aggressive, malignant primary brain tumour of adults. Tumour recurrence is all but inevitable after optimal primary treatment with maximal safe surgical resection followed by radiation \pm temozolomide (alkylating) chemotherapy. ${ }^{4}$ Options for recurrent GBM are limited, and include lomustine chemotherapy, temozolomide, reirradiation and surgical re-resection. While the optimal therapeutic paradigm for recurrent GBM is unclear, outcomes are dismal regardless of the approach and novel treatment strategies are required urgently. The cannabinoid receptors $C B_{1}$ and $C B_{2}$ are expressed in gliomas, and although the study of their expression has been limited, $\mathrm{CB}_{2}$ expression may positively correlate with tumour grade, ${ }^{5}$ although expression levels by immunohistochemistry are heterogeneous, including in grade IV (GBM) tumours. ${ }^{5,6}$ Specific cannabinoids have been investigated in numerous preclinical glioma models, where they can have anti-cancer effects, reducing tumour cell proliferation, inducing tumour cell death, and modulating angiogenesis. ${ }^{1-3}$ While temozolomide or THC have no or very minor effects on GBM cell line viability in vitro, either results in a significant reduction in tumour growth of subcutaneous xenografts of the U87MG GBM cell line in a murine model, when injected peritumourally. ${ }^{7}$ Temozolomide and THC in combination resulted in complete abrogation of xenograft growth, which appears to be mediated through autophagic stimulation (which may also have unwanted effects ${ }^{8}$ ) and apoptotic cell death. CBD and $\mathrm{THC}$ in combination resulted in significantly reduced viability of GBM cell lines and a primary human GBM culture in vitro and U87MG xenografts in vivo, with results suggesting synergy between these agents in effecting tumour cell death at submaximal concentrations, with distinct mechanisms induced by each agent, but both promoting apoptosis. Nabiximols oromucosal spray (Sativex ${ }^{\circledR}$ ) is approved in multiple countries to reduce (otherwise refractory) moderate to severe spasticity associated with multiple sclerosis, and principally comprises Cannabis sativa leaf- and flower-derived THC and CBD in an 1:1 ratio with an ethanol excipient. Similar preclinical anti-tumour effects were also seen in GBM cells with a 'Sativex-like' formulation, including significant xenograft growth inhibition in combination with temozolomide. ${ }^{7}$ This was also seen using the relatively temozolomide-resistant GBM cell line T98G, where a combination of temozolomide and THC or a 'Sativex-like' formulation led to significant tumour growth inhibition (unobserved with any of these alone). Consistent findings were later observed from similar experiments using oral cannabinoid administration, while such synergy was not observed between these compounds and lomustine chemotherapy (which is commonly used in recurrent GBM). ${ }^{9}$ These data, and other supporting findings, led to the hypotheses that THC or THC/CBD combinations (including Sative ${ }^{\circledR}$ ) in combination with temozolomide may have potential clinical utility in patients with GBM. A pilot study of intracranial THC administration directly into the surgical cavity of nine patients with recurrent, resected GBM showed a reduction in tumour cell proliferation in the two patients who had further biopsies on the study. ${ }^{6}$ While this approach appeared tolerable, outcomes in this study remained poor. Anecdotal reports and case series suggesting anti-cancer efficacy of cannabinoid preparations in patients with $\mathrm{GBM}^{10}$ are limited by possible selection bias, other concomitant or recent treatments with proven efficacy, and potential issues with pseudo-progression after such treatments.

In this issue of the British Journal of Cancer, Twelves et al. ${ }^{11}$ present the findings of their well-executed, Phase $1 \mathrm{~b}$ study of the 
tolerability and safety (primary objective) and pharmacokinetics and preliminary efficacy (secondary objectives) of nabiximols oromucosal spray (nabiximols) and dose-intense temozolomide (DIT) in 27 patients with a first recurrence of GBM following radiotherapy and temozolomide as first-line treatment. Part 1 of the study was open-label $(n=6)$ and Part 2 was randomised (1:1 to either nabiximols $(n=12)$ or placebo $(n=9))$, and doubleblind. Temozolomide was given at $85 \mathrm{mg} / \mathrm{m}^{2}$ daily (days 1-21 of each 28-days cycle with a maximum of 13 cycles), starting one week before the addition of nabiximols/placebo which was administered in an individualised manner (starting with one spray per day and building up to 3-12 sprays/day depending on tolerability, a dosing strategy based on a prior study in chronic pain $^{12}$ ).

Three patients (50\%) in Part 1, and three patients (17.6\%) in Part 2 , came off study owing to treatment-emergent adverse events (TRAEs). Four patients completed study treatment, all in Part 2 (with three in the placebo arm). The mean dose of nabiximols was 6 sprays/day (Part 1) and 7.5 sprays/day (Part 2) and the mean duration of exposure to nabiximols was 16 weeks (Part 1) and 24.9 weeks (Part 2; cf. 10 sprays/day and 23.6 weeks for placebo). The most frequent TRAEs in patients treated with nabiximols and DIT were vomiting, dizziness, nausea, fatigue and headache. These were predominantly low grade, with most being more frequent in the nabiximols arm of Part 2 (e.g. nausea in $75 \%$ versus $11.1 \%$ in patients receiving placebo). Grade 3 or greater TRAEs in patients receiving nabiximols included non-cardiac chest pain, headache, pulmonary embolism, constipation, peripheral oedema, anaemia, and urinary tract infection. There were no grade 3 or greater TRAEs in patients receiving placebo. Nabiximols had no discernible effect on temozolomide exposure.

The pre-defined efficacy endpoints were progression-free survival at 6 months (PFS6) and overall survival (OS) at 1 year for both parts of the study. PFS6 for patients in Part 1 was $16.7 \%$ (with one PFS status being unknown). PFS6 for patients in Part 2 was $33.3 \%$ for both arms of the study (with one PFS status being unknown for each arm). OS at 1 year for patients in Part 1 was $50 \%$, and in Part 2 was $83.3 \%$ for the nabiximols group and $44.4 \%$ for those receiving placebo. A log-rank test suggested a significant survival advantage in favour of nabiximols ( $p=$ 0.042 ). However, the trial was not powered to detect survival differences between groups in Part 2 (and see important caveats below). The OS at 1 year in Part 1 where all patients were receiving nabiximols was $50 \%$ (similar to the placebo group in Part 2 but these are all small numbers). In a post hoc analysis of survival (collected outside the study protocol), 2-year OS was $50 \%$ versus $22 \%$ in favour of patients treated with nabiximols ( $p=$ 0.134). Similar limitations apply to the interpretation of these findings.

This is the first study to assess the safety of nabiximols in combination with DIT, and does so in a challenging patient population. The authors have shown that dosing with nabiximols using an individualised schedule is feasible, although the incidence of TRAEs was higher than those observed previously -this could be owing to uncertainty regarding disease-related and treatment-related AEs, treatment duration, the concomitant temozolomide, or indeed a true emergent nabiximols toxicity profile for GBM patients (or a combination of these). The population enrolled was younger and had better performance status compared with most patients with recurrent GBM, and the generalisability of toxicity findings to other patients remains unclear from this small study. A significant number of patients discontinued treatment, suggesting that this is not an easily tolerated treatment combination even in this highly selected group, although this must be tempered with the caveats above and any potential therapeutic benefit in this population.
The efficacy findings in this study are intriguing but must be interpreted with significant caution, particularly given the small number of patients included in the study. This population had excellent prognosticating features: compared with the general population of patients with recurrent GBM, they were younger (mean age 50.2 years for Part 1 and 57.8 years for Part 2), fitter (with a median KPS of $90 \%$ for all groups with more patients with KPS $>/=90 \%$ in the nabiximols arm of Part 2), and with a significantly longer median time to recurrence after primary treatment-this was 20.2, 22.9 and 19.6 months from initial diagnosis for those in Part 1, Part 2 (nabiximols) and Part 2 (placebo), respectively. Importantly, the absence of (now standard) prognostic and biomarker information available within the study (e.g. IDH1/2 mutational [prognostic biomarker] and MGMT methylation status [predictive of temozolomide efficacy]) means that assessment for potential Part 2 arm enrichments/imbalances is not possible, and treatment information was not collected after study drug discontinuation/completion. Two early deaths (within 40 days) in the placebo arm of Part 2, which may simply be related to specific patient/disease factors, appear to count for much of the observed survival differences in Part 2. Additionally, there is no standard dose and schedule for DIT, the reasons for the dose and schedule in this study are unclear, and there is no evidence that DIT delivers a better outcome compared with standard temozolomide dosing schedules. ${ }^{13}$

Regardless of any potential differences between arms in Part 2, the overall survival in this trial population remains impressive, although it is difficult to conceive how this may be modulated by either nabiximols or DIT given the absence of a signal for improvement in PFS. Nevertheless, these interesting findings, backed up by a strong preclinical rationale, still warrant urgent exploration of the combination of temozolomide and nabiximols in a sufficiently powered larger study. A Phase $1 \mathrm{~b}$ study (GEINOCANN; NCT03529448) will study TN-TC11G in combination with radiotherapy and temozolomide in first-line treatment of GBM (with a recruitment target of 30 patients). It is crucial that clinical research efforts are combined with solid translational research to attempt to identify any potential predictive biomarkers for cannabinoid efficacy.

We argue against imprudent use of off-label nabiximols or other cannabinoid products in patients with GBM (which appears to be increasingly widespread and often patient-led ${ }^{14}$ ), particularly given the significant TRAE profile observed, until such time as high-quality evidence supporting or refuting the use of a specific product, in a specific population, becomes available.

\section{ACKNOWLEDGEMENTS}

Not applicable.

\section{AUTHOR CONTRIBUTIONS}

GJD designed and wrote the first draft of the manuscript. BHRdP reviewed the relevant data discussed and contributed to manuscript writing. Both authors edited the manuscript and approved the manuscript for final publication.

\section{ADDITIONAL INFORMATION}

Ethical approval and consent to participate No formal ethics approval was required for this manuscript, as per the local ethics committee and national guidance. No consent to participate was required.

Data availability Not applicable.

Competing interests The authors declare no competing interests.

Funding The authors received no specific funding for this work. 
Publisher's note Springer Nature remains neutral with regard to jurisdictional claims in published maps and institutional affiliations.

Gary J. Doherty (iD ${ }^{1,2}$ and Bruno H. R. de Paula ${ }^{1,2,3}$ 'Department of Oncology, Cambridge University Hospitals NHS Foundation, Trust, Cambridge Biomedical Campus, Cambridge, UK; ${ }^{2}$ Cancer Research UK Cambridge Centre, Cambridge, UK and ${ }^{3}$ Early Phase Trials Unit, Department of Oncology, University of Cambridge,

Cambridge, UK

Correspondence: Gary J. Doherty (gd231@cam.ac.uk)

\section{REFERENCES}

1. Lal, S., Shekher, A., Puneet, Narula, A. S., Abrahamse, H. \& Gupta, S. C. Cannabis and its constituents for cancer: history, biogenesis, chemistry and pharmacological activities. Pharm. Res. 163, 105302 (2020).

2. Śledziński, P., Zeyland, J., Słomski, R. \& Nowak, A. The current state and future perspectives of cannabinoids in cancer biology. Cancer Med. 7, 765-775 (2018).

3. Zhelyazkova, M., Kirilov, B. \& Momekov, G. The pharmacological basis for application of cannabidiol in cancer chemotherapy. Pharmacia 67, 239-252 (2020).

4. Weller, M., van den Bent, M., Preusser, M., Le Rhun, E., Tonn, J. C., Minniti, G. et al. EANO guidelines on the diagnosis and treatment of diffuse gliomas of adulthood. Nat. Rev. Clin. Oncol. https://doi.org/10.1038/s41571-020-00447-z (2020).

5. Ellert-Miklaszewska, A., Grajkowska, W., Gabrusiewicz, K., Kaminska, B. \& Konarska, L. Distinctive pattern of cannabinoid receptor type II (CB2) expression in adult and pediatric brain tumors. Brain Res. 1137, 161-169 (2007).
6. Guzmán, M., Duarte, M. J., Blázquez, C., Ravina, J., Rosa, M. C., Galve-Roperh, I. et al. A pilot clinical study of $\Delta 9$-tetrahydrocannabinol in patients with recurrent glioblastoma multiforme. Br. J. Cancer 95, 197-203 (2006).

7. Torres, S., Lorente, M., Rodríguez-Fornés, F., Hernández-Tiedra, S., Salazar, M García-Taboada, E. et al. A combined preclinical therapy of cannabinoids and temozolomide against glioma. Mol. Cancer Ther. 10, 90-103 (2011).

8. Ivanov, V. N., Grabham, P. W., Wu, C. C. \& Hei, T. K. Inhibition of autophagic flux differently modulates cannabidiol-induced death in 2D and 3D glioblastoma cell cultures. Sci. Rep. 10, 2687 (2020).

9. López-Valero, I., Torres, S., Salazar-Roa, M., García-Taboada, E., Hernández-Tiedra, S., Guzmán, M. et al. Optimization of a preclinical therapy of cannabinoids in combination with temozolomide against glioma. Biochem. Pharm. 157, 275-284 (2018).

10. Likar, R., Koestenberger, M., Stultschnig, M. \& Nahler, G. Concomitant treatment of malignant brain tumours with CBD - A case series and review of the literature. Anticancer Res. 39, 5797-5801 (2019).

11. Twelves, C., Sabel, M., Checketts, D., Miller, S., Tayo, B., Jove, M. et al. A Phase 1b randomised, placebo-controlled trial of nabiximols cannabinoid oromucosal spray with temozolomide in patients with recurrent glioblastoma. Br. J. Cancer https://doi.org/10.1038/s41416-021-01259-3 (2021).

12. Ballman, K. V., Buckner, J. C., Brown, P. D., Giannini, C., Flynn, P. J., LaPlant, B. R. et al. The relationship between six-month progression-free survival and 12 month overall survival end points for phase II trials in patients with glioblastoma multiforme. Neuro Oncol. 9, 29-38 (2007).

13. Van Den Bent, M. J. \& Taal, W. Are we done with dose-intense temozolomide in recurrent glioblastoma? Neuro Oncol. 16, 1161-1163 (2014).

14. Reblin, M., Sahebjam, S., Peeri, N. C., Martinez, Y. C., Thompson, Z. \& Egan, K. M. Medical cannabis use in glioma patients treated at a comprehensive cancer center in florida. J. Palliat. Med. 22, 1202-1207 (2019). 